Stephanie Jacobs*

\title{
Zeichen - Bücher - Netze: Das Deutsche Buch- und Schriftmuseum der Deutschen Nationalbibliothek
}

\author{
Ein Ort der Buch- und Mediengeschichte
}

DOI 10.1515/bfp-2017-0005

Zusammenfassung: Die Sammlung, Ausstellung und wissenschaftliche Bearbeitung buch- und mediengeschichtlicher Zeugnisse ist die Aufgabe des Deutschen Buch- und Schriftmuseums. Im Fokus der Arbeit steht das Buch mit seinen zahllosen Gesichtern und Funktionen - auch nach dem Übergang ins Zeitalter der digitalen Netzwelt. Einerseits wissenschaftliche Dokumentationsstätte für Buchkultur und Mediengeschichte lädt das Museum andererseits mit seinen Ausstellungen und museumspädagogischen Programmen als ein lebendiger Ort kultureller Bildung und Vermittlung zum Verweilen ein.

Schlüsselwörter: Buchmuseum; Mediengeschichte; Ausstellung; virtuelle Ausstellung; Leipzig

\section{Signs - Books - Networks: German Museum of Books and Writing of the German National Library. A place of the History of Books and Media}

\begin{abstract}
The task of the German Museum of Books and Writing is to collect, exhibit, and process evidence of book and media history. The main focus of the museum's work is on the book and its myriad aspects, even after the transition to the era of digital networks. It is a museum which acts as an academic documentation centre for book and media history. But it is also an inviting and vibrant place of cultural education, aimed at bringing culture to a wider audience and attracting visitors with its exhibitions and educational programmes.
\end{abstract}

Keywords: Museum of Books and Writing; history of media; exposition; virtual exposition; Leipzig

Inhalt

1 Prolog....................... 202

2 Ein neues Haus für ein altes Museum: Zur

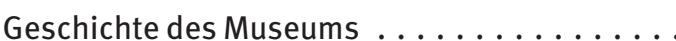

*Kontaktperson: Dr. Stephanie Jacobs, s.jacobs@dnb.de
3 Buchmuseen am Anfang des 21. Jahrhunderts Aufgaben und gesellschaftliche Verantwortung . . 204

4 Die Bestände. . . . . . . . . . . . . . . . . 206

5 „Zeichen - Bücher - Netze. Von der Keilschrift zum Binärcode“ Die neue Dauerausstellung . . . . 207

6 Mediengeschichte im Netz: Die virtuelle Ausstellung des Deutschen Buch- und Schriftmuseums . . . . . . . . . . . . . . . . . . . 209

7 Die Wechselausstellungen . . . . . . . . . . 210

\section{Prolog}

Wenn das Deutsche Buch- und Schriftmuseum der Deutschen Nationalbibliothek ${ }^{1}$ im Frühjahr 2016 eine Ausstellung unter dem Titel „Bahnriss?! Papier|Kultur“2 eröffnet, die die Papierkultur und deren prognostiziertes Ende befragt, dann gibt das Museum seinen Besuchern nicht nur einen Einblick in seine einzigartigen Sammlungen zur Papiergeschichte, sondern stellt sich auch einer aktuellen gesellschaftlichen Fragestellung: Seit Jahren ist das Papier nicht mehr selbstverständlicher Träger von Informationen. Die digitalen Medien, die in vielerlei Hinsicht das effizientere Verbreitungsmedium sind, haben im Alltag dem Papier als jahrtausendealtem Beschreibstoff den Rang abgelaufen. Wie sieht die Zukunft des Papiers aus? Wird die Papierbahn ganz reißen? Welche Bedeutung hat dieser „Bahnriss“ für unsere Kultur und deren Instrumente? Und: Was passiert mit den Orten des Papiers - den Bibliotheken, Museen und Archiven? Denn spätestens seitdem der Text nicht mehr an die schwarz-auf-weiß gedruckte und zwischen zwei Buchdeckeln eingefangene Seite gebunden, sondern über digitale Netze weltweit abrufbar ist, steht das Selbstverständnis der Bibliothek und des Archivs als

1 Vgl. zuletzt Jacobs (2014b). Einzelne Themen und Abschnitte des vorliegenden Aufsatzes wurden hier bereits veröffentlicht. Vgl. allgemein zu den Aufgaben des Deutschen Buch- und Schriftmuseums der Deutschen Nationalbibliothek auch Fabian (1994).

2 Schmidt (2016). 


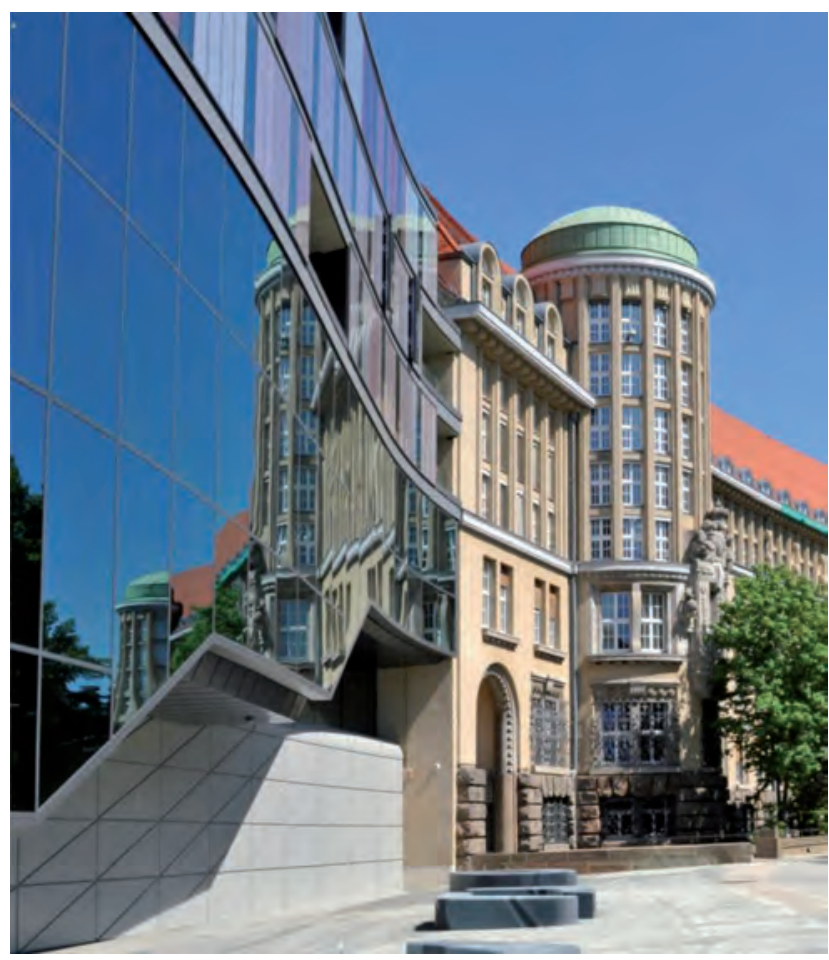

Abb. 1: EB Front: Das Deutsche Buch- und Schriftmuseum im Erweiterungsbau der Deutschen Nationalbibliothek, Leipzig. @ Deutsche Nationalbibliothek, Bärbel Kaiser

Orte der Information, als Wissensquelle mit Alleinvertretungsanspruch zur Disposition.

Mit ihrem Blick auf aktuelle Fragestellungen ist die Ausstellung „Bahnriss?! Papier|Kultur“ symptomatisch für das Themenspektrum des Deutschen Buch- und Schriftmuseums: Gegenwart aus der Geschichte heraus zu beleuchten und zu verstehen, aktuelle Fragestellungen an den mehr als eine Million Bestände umfassenden Sammlungen des Museums zur Buch- und Mediengeschichte abzuprüfen und Artefakte aus der Buchgeschichte neuen Fragestellungen auszusetzen, das ist das Ziel der musealen Arbeit. Das Museum versteht sich nicht nur als Hort nostalgischer Erfahrungen, sondern auch als Diskursraum für einen mit historischem Weitblick und Gelassenheit geschulten Blick in die Zukunft der Medien.

\section{Ein neues Haus für ein altes Museum: Zur Geschichte des Museums}

Für das 1884 in der damaligen Buchstadt Leipzig gegründete Deutsche Buch- und Schriftmuseum beginnt im Jahr 2012 eine neue Ära: Das Museum bekommt einen Neubau, in dem nicht nur seine Sammlungen eine neue Heimstatt unter optimalen konservatorischen Bedingungen bekommen, sondern auch eine neue Dauerausstellung eröffnet werden konnte. Nach über 60 Jahren der interimistischen Unterbringung am Deutschen Platz können die über eine Million Bestandseinheiten zählenden Sammlungen erstmals geschlossen aufgestellt werden. Die neuen Ausstellungsflächen im Erdgeschoss des Erweiterungsbaus bieten dem Museum umfassende Präsentationsmöglichkeiten, die Museumspädagogik erhält einen prominent gelegenen Raum im Foyerbereich und die Mitarbeiter des Museums beziehen gemeinsame Räume im Neubau.

Für das Deutsche Buch- und Schriftmuseum ist der Bau, für den in einem mehrstufigen Wettbewerb der metaphorische Entwurf „Umschlag - Hülle - Inhalt“ der Stuttgarter Architektin Gabriele Glöckler aus Stuttgart ausgewählt wurde, ein Glücksfall. Das zeigt auch ein kurzer Blick in die Geschichte des Museums ${ }^{3}$ : Das Ende des 19. Jahrhunderts gegründete und nach Qualität und Umfang seiner Bestände eines der weltweit bedeutendsten Buchmuseen ist in einer mediengeschichtlich komplexen und unruhigen Zeit als Gewerbemuseum konzipiert worden. Spätestens 1914, als das Museum umfangreiche Bestände aus der in Leipzig veranstalteten „Internationalen Ausstellung für Buchgewerbe und Graphik“ (Bugra) übernehmen kann, erweitert das Museum seinen Horizont über den engen Rahmen eines gewerblichen Museums hin zu einer Institution, die die Mediengeschichte insgesamt in den Blick nimmt. Es thematisiert die Geschichte der Schrift - auch die außereuropäischer Kulturen - ebenso wie die Papiergeschichte, die Geschichte der Reproduktionstechniken und die der wissenschaftlichen Grafik.

Doch reißt der Erste Weltkrieg und die folgenden, durch Inflation und politisch-gesellschaftliche Umbrüche gezeichneten Jahre das vor 1914 prosperierende Museum in eine finanzielle Krise. Als ultima ratio plant der Sächsische Staat 1922, das teuerste Museumsobjekt, eine zweibändige Pergamentausgabe der Gutenberg-Bibel, zu verkaufen. Der Erlös soll zur Deckung der Finanzierungslücken für ausstehende Mieten, Löhne und Heizungskosten dienen. Um diesen Verkauf zu verhindern, gelingt es dem Direktor des Museums, Hans-Heinrich Bockwitz, Künstler dazu zu animieren, zugunsten des Museums eine Grapikmappe aufzulegen - unter ihnen Paul Klee, Oskar Kokoschka, Alfred Kubin, Lovis Corinth und Käthe Kollwitz. Die Aktion, die als „Künstlerspende“ in die Annalen des Museums eingegangen ist, verläuft so erfolgreich, dass der Verkauf der Gutenberg-Bibel verhindert werden kann.

3 Chronik des Museums unter http://www.dnb.de/DE/DBSM/Chronik/chronik_node.html. Vgl. auch Funke (1984). 
Schwere Verluste erleiden Museum und Sammlung im Zweiten Weltkrieg: Das Buchgewerbehaus - Sitz des Museums seit 1939 - wird in der Nacht vom 3. auf den 4. Dezember 1943 in Schutt und Asche gelegt. Das Museum verliert laut Meldungen des Kriegsschadensamtes von September 1944 allein neun Zehntel seines Buchbestandes. Die wertvollsten, damals auf Schloss Rauenstein im Erzgebirge ausgelagerten Stücke der Klemm-Sammlung Handschriften, Inkunabeln (darunter die 42-zeilige Gutenberg-Bibel) und Drucke aus dem 16. bis 19. Jahrhundert, eine Einband- und eine Zeugdrucksammlung - beschlagnahmt die sowjetische Besatzungsmacht im September 1945 und verbringt sie nach Moskau, wo sie noch heute in der Russischen Staatsbibliothek liegen. ${ }^{4}$

Nach dem kriegsbedingten Verlust seiner Wirkungsstätte wird das Museum 1950 in die Räume der Deutschen Bücherei integriert und erhält dadurch die Chance zu einem Neuanfang. Dank eines gesicherten, wenngleich bescheidenen Ankaufsetats kann mit dem Wiederaufbau der Sammlungen begonnen werden. In den folgenden Jahrzehnten wird eine Konsolidierung des Museums erreicht, die räumliche Unterbringung seiner Bestände und Ausstellungsflächen jedoch setzen der Entwicklungsperspektive enge Grenzen.

Mit der Auslobung des Architekturwettbewerbs für die Deutsche Nationalbibliothek 2002 rückt endlich in greifbare Nähe, was bereits am Anfang des 20. Jahrhunderts für das Museum als notwendig erachtet worden war, aber durch den Ausbruch des Ersten Weltkrieges vereitelt wurde: ein Museumsneubau. In konzeptionell-thematischer Hinsicht sind die musealen Neubauplanungen am Anfang des 21. Jahrhunderts ein mutiger Schritt, denn die buchhistorisch ausgerichtete Institution bekommt ausgerechnet zu einer Zeit, in der die Diskussion um die Zukunft des Buches in breiter Öffentlichkeit geführt wird, wieder die Räume und die städtebauliche Präsenz, die es zuletzt in der ersten Hälfte des 20. Jahrhunderts hatte. Am Anfang des 21. Jahrhunderts der Geschichte des Buches und der Medien eine Bühne zu schaffen, ist ein klares Bekenntnis zur Aufgabe des Museums: aktuelle Debatten anzustoßen und sich nicht auf die Konservierung des Historischen zurückzuziehen. Dieser Verantwortung - in Wissenschaft, Bildungsarbeit und Kultur - gerecht zu werden, ist die Messlatte für die museale Arbeit.

Angesichts seiner neuen Architektur und der sich rasant verändernden Buch- und Medienwelt stellt sich für das Museum also vor allem eine Frage: Ist ein neues Buchmuseum heute nicht ein nostalgischer Anachronismus?

4 Poethe (1997), Jacobs (2014a) sowie Jacobs und Rüdiger (2016).

\section{Buchmuseen am Anfang des 21. Jahrhunderts - Aufgaben und gesellschaftliche Verantwortung}

Das Buch war jahrhundertelang der erfolgreichste Wissensspeicher. Aber nicht erst mit dem Siegeszug der elektronischen Wissensvernetzung ist das Buch in die Defensive geraten. Bereits durch den Film, das Radio und das Fernsehen hat es an Territorium eingebüßt, zugleich aber immer wieder an Wachstum gewonnen, indem es Kulturbereiche für sich eroberte oder solche besetzt hielt, in denen das Buch das beste Medium der Informationsvermittlung war.

Ist aber nun - mit E-Book, E-Paper und E-Tinte - die Krise des Buches endlich da, die die Kulturpessimisten seit vielen Jahrzehnten heraufbeschwören? Vom Standpunkt der Alleinvertretung für das Weltwissen ist das Buch in einer Krise, von der es sich auch nicht wieder erholt. Aber diese Krise bedeutet zugleich einen Zugewinn an Kultur, Wissenschaft und Bildung: Um wie viel schneller und präziser ist der Informationstransfer geworden? Wieviel demokratisches Potenzial liegt in den digitalen Netzen? Im Hinblick auf den schnellen Austausch von Wissen und dessen Verfügbarkeit ist der gedruckte Text schon lange nicht mehr konkurrenzfähig. Was aber sind in diesem gesellschaftlichen und technischen Umbruch das Thema und die bildungspolitische Verantwortung eines Buchmuseums?

Es liegt nahe, den gedruckten Text und den Kodex als zwei Jahrtausend-Erfindungen noch einmal zu feiern, das Buch im Museum feierlich zu Grabe zu tragen - entweder nostalgisch rückwärtsgewandt oder ästhetisch überhöhend. Ist das Museum nicht der Ort der Nostalgie, der Rückbesinnung und Einkehr, den weder Urheberrechtsquerelen noch die Unkenrufe des Buchmarktes erreichen, vor dem der unruhestiftende mediengeschichtliche Umbruch halt macht?

Das klingt naheliegend, wäre aber das Ende des Museums als Ort von Bildung und Aufklärung. Zwar speichert und bewahrt das Museum Vergangenheiten, jedoch nicht um ihrer selbst willen, sondern für einen Abgleich mit der Gegenwart, die sich an den Hinterlassenschaften der Geschichte vergewissert, historische Lösungsstrategien kennenlernt und daraus Visionen für die Zukunft entwickelt. In diesem Sinne zielt das Deutsche Buch- und Schriftmuseum mit seiner Neupositionierung auf kulturelle Verantwortung sowohl gegenüber seinem Gegenstand als auch gegenüber den Fragestellungen der heutigen Gesellschaft. Die Unfähigkeit zur Gegenwart, die der Institution Museum immer wieder vorgeworfen wird, ist keine Zwangsläufigkeit.

Aus der gesellschaftlichen Verantwortung von $\mathrm{Mu}-$ seen ergeben sich drei Handlungsstränge und drei unter- 


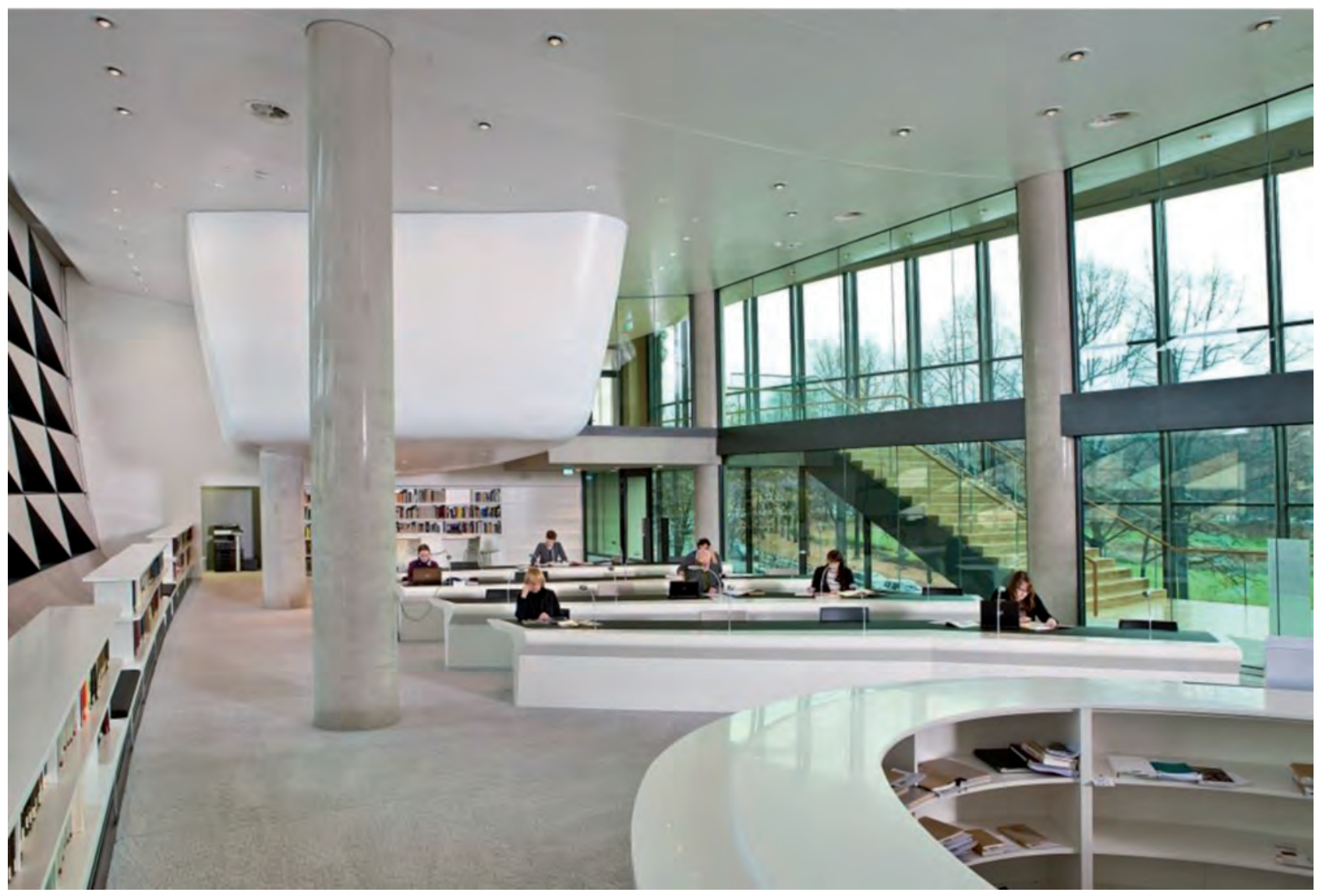

Abb. 2: Museumslesesaal im Erweiterungsbau der Deutschen Nationalbibliothek. @ Kunstmann

schiedliche Adressatenkreise für unsere Arbeit an der Buch- und Mediengeschichte, denen gegenüber wir eine Bringschuld haben: die Fachcommunity, die Kinder und Jugendlichen, deren Ausbildung unsere Zukunft sichert, und eine interessierte, breite Öffentlichkeit:

Fachcommunity: Das Deutsche Buch- und Schriftmuseum ist zentrale wissenschaftliche Dokumentationsstätte für die Buchkultur in Deutschland, das seine Bestände aus 5000 Jahren Schrift-, Buch-, Medien- und Papiergeschichte Wissenschaftlern zur Verfügung stellt und an Studierende vermittelt. Zentraler Ort für diese Aufgabe als Forschungsstätte sind neben dem World Wide Web und dem elektronischen Katalog der Deutschen Nationalbibliothek, in dem die Bestände des Deutschen Buch- und Schriftmuseums (noch nicht vollständig) verzeichnet sind, der neue Lesesaal und der Seminarraum des Museums. Neben 85000 Bänden Fachliteratur und 200 laufenden Zeitschriften können hier eine umfangreiche Sammlung von Archivalien zur Buchgeschichte, einzigartige papierhistorische Bestände mit der weltweit größten Wasserzeichensammlung, eine Sammlung an Frühdrucken, Künstlerbücher, grafische Blätter und Plakate zur Mediengeschichte und andere historische Dokumente für die Forschung bereitgestellt werden.

Kinder und Jugendliche: Das Museum ist Bildungsinstitution und außerschulischer Lernort, an dem Neugier und Lernen, Vergnügen, Unterhaltung und Bildung keine Widersprüche sind und an dem historische Zeugnisse Geschichte anschaulich und begreifbar machen. Dies ist besonders wichtig in einer Zeit, in der einerseits die allgegenwärtige Verfügbarkeit von Wissen im Netz den Sinn für die Authentizität von Originalobjekten schärft, und in der andererseits die Frage nach integrativer Bildung und Wissensvermittlung an Menschen aus anderen Kulturkreisen immer wichtiger wird. Der Ort für die museumspädagogische Arbeit ist das Museumskabinett, das direkt vom Museumsfoyer aus zugänglich ist.

Kulturinteressierte Öffentlichkeit: Das Museum ist aber auch eine „Schaubude“ - ein antiquierter, aber treffender Begriff des 19. Jahrhunderts, den Peter Sloterdijk reaktiviert hat: das Museum als ein Raum für bücherliebende Augenmenschen und als eine Schule des Sehens, in der Wissen vor allem über den Augensinn rezipiert wird. Orte dieser „Schaubude“, die auch Unterhaltung, Vergnügen und intellektuelle Anregung vermitteln sollen, sind die 


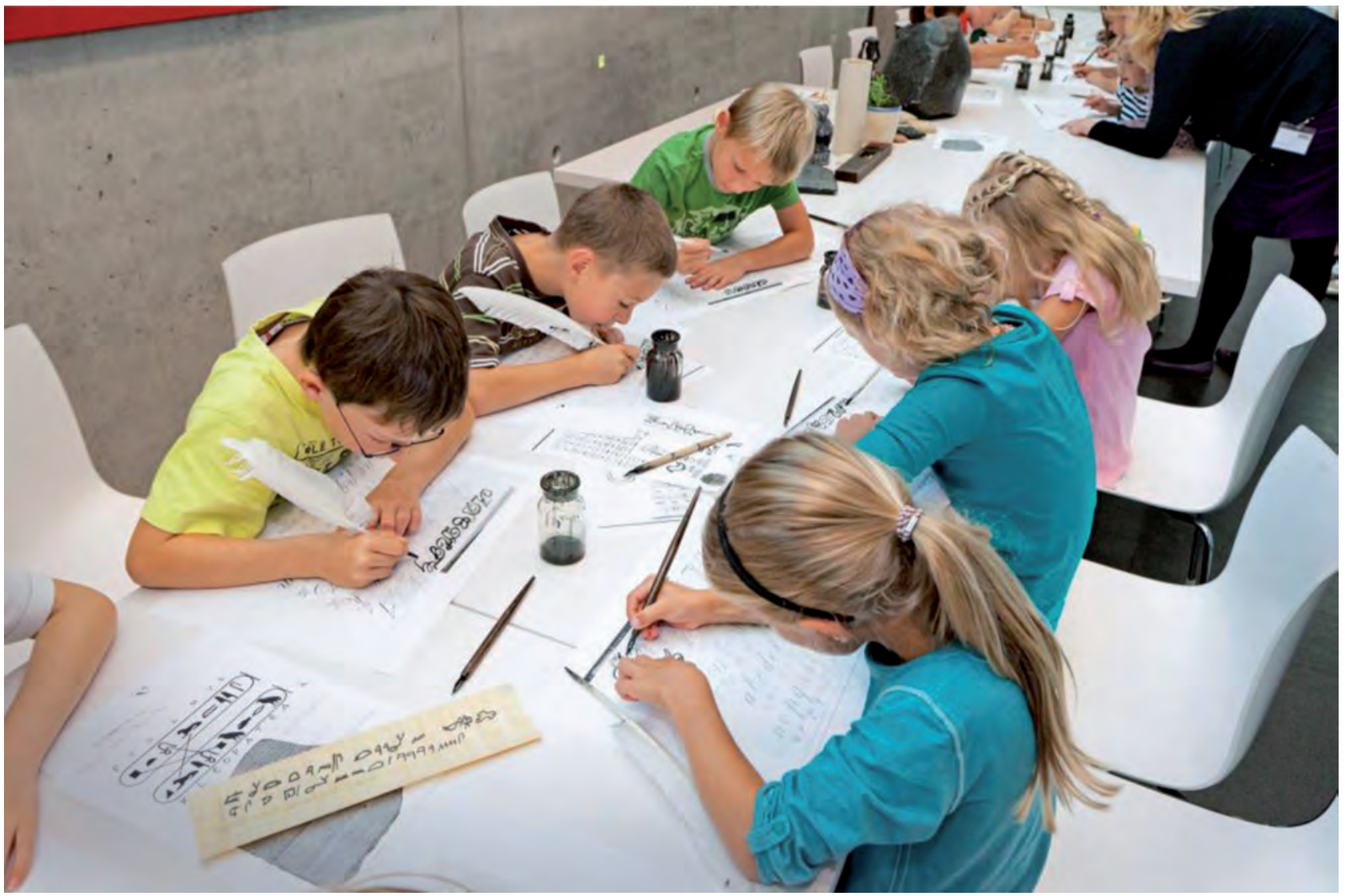

Abb. 3: Arbeit mit Kindern im Museumskabinett. ${ }^{@}$ Klaus-D. Sonntag

Ausstellungsräume des Museums: die neue Dauerausstellung, die unter dem Titel „Zeichen - Bücher - Netze. Von der Keilschrift zum Binärcode“ Einblick in 5000 Jahre Mediengeschichte gibt, die schöne und außergewöhnliche, skurrile oder semantisch sperrige historische Exponate zeigt und in unterschiedlichen kulturgeschichtlichen Kontexten aufschlüsselt. Neben der ca. $1000 \mathrm{~m}^{2}$ großen Dauerausstellungshalle bieten der Wechselausstellungsraum und der Schautresor Möglichkeiten für temporäre Ausstellungen.

\section{Die Bestände}

Welche der benannten Aufgaben das Museum auch immer in den Blick nimmt: Es sind seine historischen Sammlungen, die die Arbeit erst ermöglichen, prägen und zukunftssicher machen.

Die Bestände des Deutschen Buch- und Schriftmuse$u s^{5}$ umfassen mehr als eine Million Einheiten und zeich-

5 http://www.dnb.de/DE/DBSM/Bestaende/bestaende_node.html. nen sich durch eine Heterogenität aus, die einen interdisziplinären Zugang zu Fragen der Buch-, Papier- und Mediengeschichte bieten. Den Grundstock legte die Sammlung des Dresdner Schneiders und Modeverlegers Heinrich Klemm, der 1819 in Dresden geboren wurde. Seine Sammlung buchhistorischer Muster und Fachliteratur, das „Bibliographische Museum“, wurde in seinem Todesjahr 1886 vom Sächsischen Staat für das Museum angekauft und stellt den Kern unserer Bestände dar. Inzwischen umfasst die sog. Klemmsammlung 23000 Drucke von der Inkunabelzeit bis zum 21. Jahrhundert.

Mit einem Bestand von 35000 Veröffentlichungen (museale Drucke und Fachliteratur) gibt die Sammlung Künstlerische Drucke einen Überblick über die Entwicklung der Buchgestaltung im 20. und 21. Jahrhundert.

Die Sammlung Archivalien und Dokumente zur Buchgeschichte beherbergt rund 150000 schriftliche und bildliche Quellen - insbesondere zum Buchhandel und zum Buchdruck des 16. bis 21. Jahrhunderts. Den Kern bildet das „Buchhandelsarchiv der Bibliothek des Börsenvereins der Deutschen Buchhändler zu Leipzig“, das seit 1844 vor allem deutschsprachige Archivalien zur Buchgeschichte aus dem 19. Jahrhundert systematisch sammelt. 


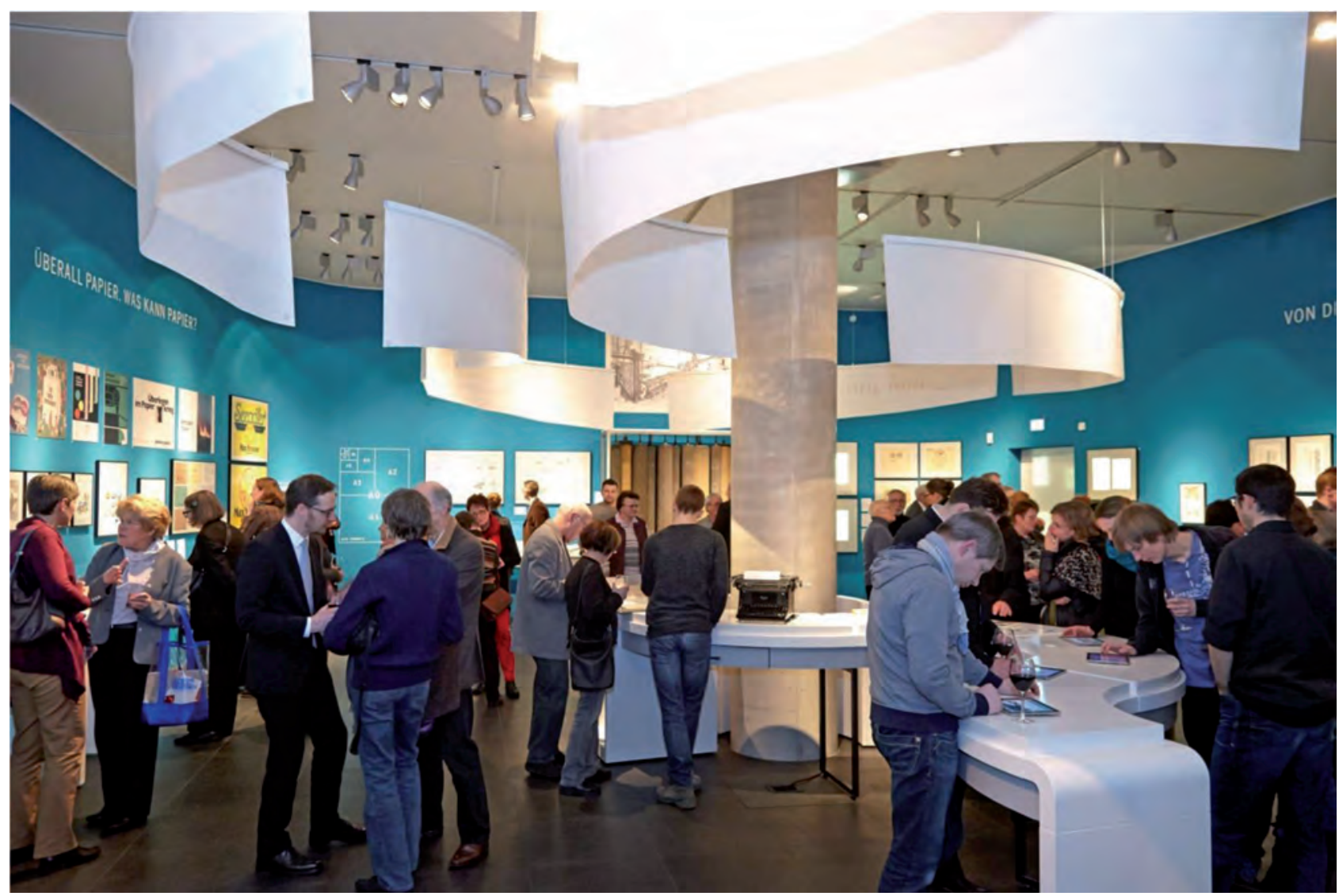

Abb.4: Eröffnung der Ausstellung „Bahnriss?! Papier|Kultur“. @ Punctum / Alexander Schmidt

Die Papierhistorischen Sammlungen umfassen $\mathrm{Pa}$ piere aus vorindustrieller Zeit und fabrikmäßiger Produktion sowie aus kunstgewerblicher und künstlerischer Fertigung. Sie stammen vor allem aus Europa, aber auch aus Asien und Amerika. Die Wasserzeichensammlung umfasst mit ca. 400000 Exemplaren die weltweit größte Sammlung dieser Art.

Die Grafische Sammlung enthält 30000 originalund reproduktionsgrafische Einzelblätter und Blattfolgen vom 15. bis zum 21. Jahrhundert. Sie bietet eine Ergänzung zum musealen Buchbestand und zielt thematisch auf gestalterische, illustrativ-künstlerische und drucktechnische Aspekte der Buch- und Schriftgeschichte.

Ferner nennt das Museum eine Kulturhistorische Sammlung sein eigen, in der neben Druckmaschinen, Geräten zur Papierherstellung und Schreibgeräten auch frühe Buchformen und Zeugnisse aus der Frühgeschichte der Schrift bewahrt werden.

Vor- und Nachlässe sind dem Museum u.a. von folgenden Buch- und Schriftkünstlern sowie Buchwissenschaftlern anvertraut worden: Axel Bertram, Karl-Heinz Birkner, Johann Goldfriedrich, Erich Gruner, Albert Kapr,
Hans Ticha, Walter Tiemann, Jan Tschichold, Hans-Peter Willberg und Hans-Joachim Walch.

Die historisch gewachsenen Sammlungen des Deutschen Buch- und Schriftmuseums sind ein gewichtiges Pfund und wertvolles Erbe, sie sind eine Verpflichtung für die Zukunft. Ihre Vernetzung mit der wissenschaftlichen Gemeinschaft, ihr Einsatz in der Ausstellungs- und Bildungsarbeit sind das Ziel der Museumsarbeit. Sie sind Wunderkammer und Zukunftswerkstatt zugleich.

\section{5 „Zeichen - Bücher - Netze. Von der Keilschrift zum Binärcode“ Die neue Dauerausstellung}

Mit der neuen Dauerausstellung ${ }^{6}$ etabliert das Museum in der offenen Architektur des Erweiterungsbaus ein neues Ausstellungskonzept.

6 Jacobs (2016), zur Dauerausstellung vgl. auch Hintermeier (2012), Müller (2012) und Güntner (2012). 


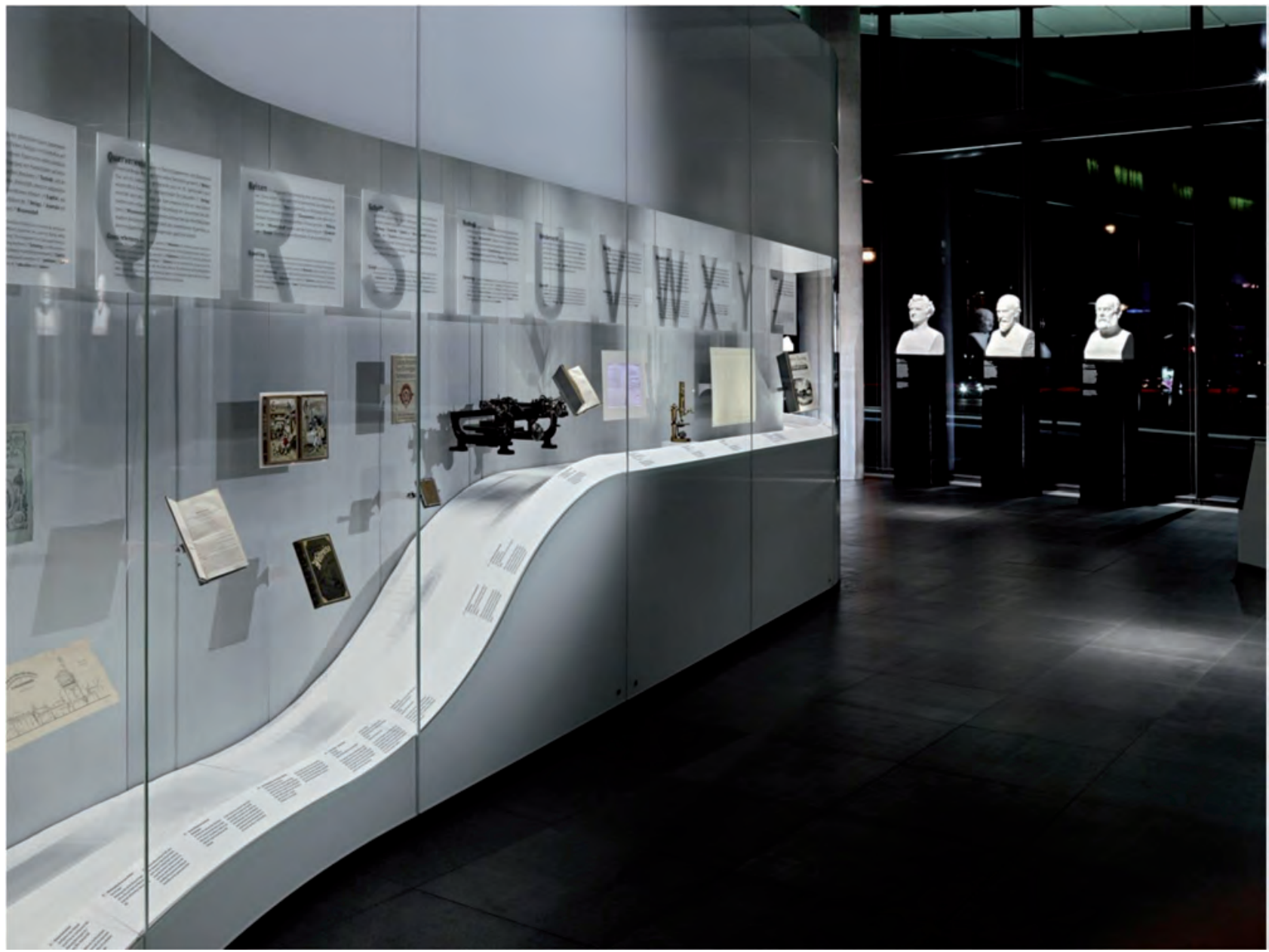

Abb. 5: Blick in die Dauerausstellung des Deutschen Buch- und Schriftmuseums der Deutschen Nationalbibliothek. ${ }^{@}$ Punctum/Bertram Kober

Die Halle, deren Offenheit dem Topos des Buchmuseums als introvertiertes Schatzkästchen schon in der äußeren Gestalt widerspricht, ist eher öffentlicher Platz als pseudosakrale Gegenwelt. Wie das Ausstellungskonzept soll auch die lichte Architektur der Dauerausstellung den Besucher mit „offenen Armen“ empfangen. Der landläufigen Vorstellung vom Museum als einem Ort, an dem Geschichte verkapselt und vor den Einflüssen der Gegenwart geschützt aufbewahrt wird, tritt ein offener Kommunikationsraum entgegen, an dem Geschichte mit Gegenwart und Zukunft verhandelt, an dem die Herausforderungen der Gegenwart an historischen Lösungen geprüft werden und an dem die Besucher über historische Zusammenhänge ins Gespräch kommen.

Modular im Aufbau kombiniert die Ausstellungskonzeption einen chronologischen Faden mit Querschnittsthemen. Durch den Weitwinkel einer kurzen Mediengeschichte der vergangenen 5000 Jahre fasst der offene Parcours durch die Ausstellungshalle die Aktualität der drei Medieninnovationen - die Erfindung der Schrift, den
Buchdruck mit beweglichen Lettern und die digitalen Netze - in einem großen zeitlichen Bogen zusammen. Aktuelle Fragestellungen knüpfen sich an historische Artefakte und fördern dadurch bisweilen erstaunliche Parallelen zwischen den historischen Innovationsschritten zutage.

Beginnend mit dem Übergang von der mündlichen in die schriftliche Kultur wird am Anfang der Ausstellung der Innovationsschub hervorgehoben, den die Erfindung der Schrift für die Menschheit bedeutet. Die Schrift hat als erstes Speichermedium die menschliche Kommunikation grundlegend verändert. Der entscheidende Vorteil des neuen Speichermediums war neben der Verlässlichkeit der fixierten Information (gegenüber der mündlichen, sich stets verändernden Überlieferung) vor allem die raum-zeitliche Abstraktion: Schriftlich übermitteltes Wissen war unabhängig von Ort und Zeit der Aufzeichnung. Und genau hier setzt auch die Kritik an der Schrift als der ersten Medieninnovation der Menschheit an: Wenn Platon in seinem Dialog Phaidros den Leser davor warnt, Schrift zu 
gebrauchen, erscheint das angesichts der Erfolgsgeschichte gerade der Alphabetschriften über tausende von Jahren ein wunderlicher Rat zu sein, denn Platons Philosophie hätte ohne die schriftliche Überlieferung niemals die Verbreitung gefunden, die ihr im Laufe der Überlieferungsgeschichte zuteilwurde.

Doch seine Begründung für das Verdikt der Schrift trifft den Kern der Datenspeicherung: Während das gesprochene Wort jederzeit vom Sprecher selbst bewertet, erläutert und kommentiert werden kann, gewinnt das geschriebene Wort durch seine Speicherung eine eigene, nicht mehr kontrollierbare Wirkmacht. Es verselbstständigt sich - ganz unabhängig davon, ob es in Ton geritzt, in Lehm gepunzt, auf Papier geschrieben, im Buch gedruckt oder als digitale Wolke auftritt: Einmal in der Welt hat die Schrift unabhängig von ihrem Autor ein zeit-räumlich unabhängiges Dasein. Das heißt, so Platon, dass die Schrift auch diejenigen erreicht, die sie nicht (richtig) verstehen oder sie missbrauchen. Außerdem entlaste die Schrift das Gedächtnis, weil dieses nicht mehr geschult werde und mache den Menschen daher dumm und vergesslich.

Die bei Platon geäußerte Skepsis gegenüber Speichermedien verbindet die Menschheitsgeschichte und ihre Medieninnovationen über Jahrtausende miteinander. Der Index librorum prohibitorum, die von der katholischen Kirche im 16. Jahrhundert aufgelegte Indexliste mit verbotenen Büchern, die in den folgenden viereinhalb Jahrhunderten immer wieder neue Auflagen erlebt hat, ist eine direkte Folge aus der neuen Technologie des Buchdrucks mit beweglichen Lettern - der zweiten Medieninnovation. Erst die durch den Buchdruck mit beweglichen Lettern ermöglichte Optimierung der Speicher- und Vervielfältigungsprozesse gibt dem gedruckten Wort seine Verbreitungsmöglichkeiten. Die Erfolgsgeschichte des Buchdrucks, die rasante Verbreitung von Informationen war aus der neuzeitlichen Gesellschaft nicht mehr wegzudenken. Der Index war ein Jahrhunderte andauernder, letztlich aber erfolgloser Versuch, das kirchliche Informationsmonopol gegen Informationsflut und freie Meinungsäußerung zu schützen. Auch hinter dem Index steckt das Misstrauen gegen die Speicherung von Wissen und die Einsicht in die Wirksamkeit des gedruckten Wortes.

Die heutige Kritik an der täglich unüberschaubarer werdenden Flut an Daten im Netz greift den Gedanken von der Massenhaftigkeit, Manipulierbarkeit und Unübersichtlichkeit der Informationen auf - das Thema der dritten digitalen - Innovation der Speicherprozesse, die die Weltgesellschaft derzeit vor große Herausforderungen stellt.

An diesem Gedankenkreis setzt die neue Dauerausstellung mit ihren elf Themenmodulen an. Ihre Titel: Laute-Zeichen-Schriften, Zeichen setzen, SchriftGestalten,
Handschriftenkultur, Buchdruck, Lesewelten, Zensur, Industrialisierung, Ästhetik des Buches, Massenmedien und Medienzukünfte. Die Ausstellung stellt Fragen und möchte den Besucher zu einer eigenen Position herausfordern: Was bedeutet es für unsere Kultur, seit der Erfindung der Schrift immer größere Informationsmengen speichern zu können? Was heißt es, wenn wir sagen, dass der Druck mit beweglichen Lettern unsere Kultur mehr verändert hat als die Erfindung von Rad und Schießpulver - wie Georg Christoph Lichtenberg sagt. Im Lichte dieser genauso einfachen wie fundamentalen Fragen setzen wir die Geschichte von Buch und Schrift neuen Ansichten aus.

\section{Mediengeschichte im Netz: Die virtuelle Ausstellung des Deutschen Buch- und Schriftmuseums}

Eine Ausstellung, die sich mit der Geschichte, Gegenwart und Zukunft der Medien beschäftigt, hat durch Einsatz moderner digitaler Technologien in besonderer Weise die Chance, ihre Themen auch in die digitalen Netze zu speisen. Daher hat das Museum ergänzend zur Dauerausstellung eine virtuelle Ausstellung erarbeitet, in der die mediengeschichtlichen Themen in einem Wissensnetz aufbereitet und interaktiv angeboten werden: ${ }^{7}$ Sei es in der Ausstellung auf mitgebrachten oder bereitliegenden mobilen Endgeräten oder als digitales Angebot im Internet, das überall abgerufen werden kann.

Ziel der multimedialen Anwendung ist es, den Besucher auf eine virtuelle Zeitreise durch die Geschichte der Medien mitzunehmen. Ausgewählte Akteure der Mediengeschichte begleiten den Besucher durch die Zeit, knapp und pointiert erzählte Objektgeschichten und historische Ereignisse werden zu einem Netz verknüpft, durch das der Besucher sich explorativ und frei von einer festgelegten Reihenfolge „schmökernd“ bewegen kann. Interaktive Karten sorgen für weitergehende topografische Hintergrundinformationen. Anhand von digitalen Texten und Bildern, Filmen und Hörbeiträgen können Online-Besucher verschiedenen thematischen Pfaden und deren chronologischer Verortung folgen.

7 http://mediengeschichte.dnb.de. 


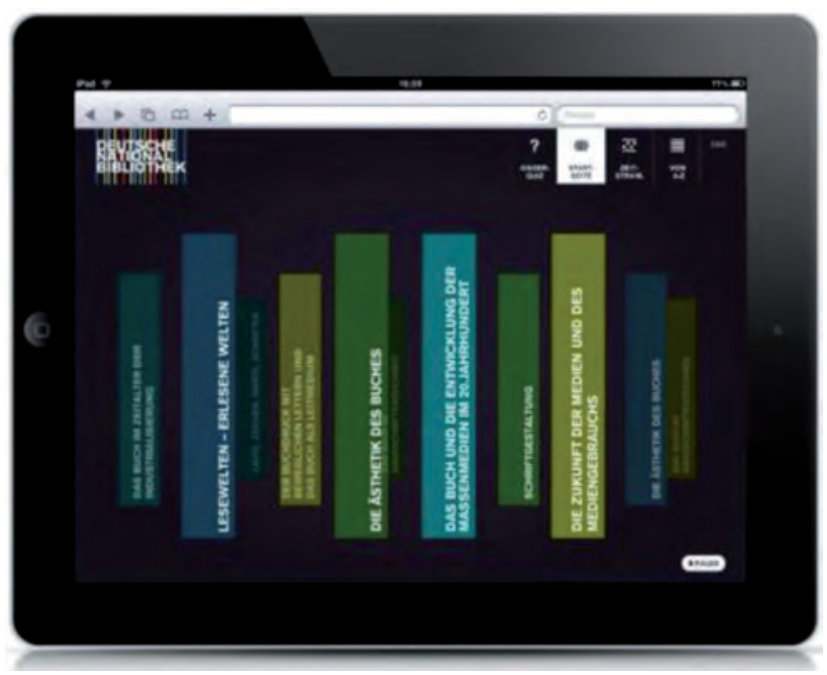

Abb. 6 Startseite der virtuellen Ausstellung des Deutschen Buch- und Schriftmuseums der Deutschen Nationalbibliothek zu 5000 Jahre Mediengeschichte ${ }^{8}$

Hinterlegt ist die virtuelle Ausstellung mit einem Glossar, das mit über 1000 Einträgen in Twitter-Kürze Erläuterungen bietet. Das Glossar schafft nicht nur Orientierung, sondern hilft auch, unterschiedliche Wissensstände beim Besucher zu berücksichtigen. Ein Medien-Quiz stärkt den interaktiven Charakter der Anwendung und lockt auch die nachwachsenden Besucher an.

Schließlich sorgt ein digitales Besucherbuch dafür, dass der Besucher direkt mit den Kuratoren ins Gespräch kommen kann und in das Ausstellungsgeschehen einbezogen ist.

\section{Die Wechselausstellungen}

Das Wechselausstellungsprogramm des Deutschen Buchund Schriftmuseums ${ }^{9}$ zielt auf eine Mischung von Themen, die einerseits aktuelle Debatten in Wissenschaft und Gesellschaft aufgreifen, andererseits aber auch durch gegen den Strich gebürstete Fragestellungen und „schräge“ Blickwinkel überraschen und Thesen zuspitzen. Darüber hinaus geht es bei dem Ausstellungsprogramm auch darum, besondere Bestände aus den Sammlungen zu präsentieren. Da die zentralen Themen des Museums - die Geschichte des Buches, der Medien, des Papiers und der Speicherung von Wissen - im besten Sinne „Allerweltsthemen" sind, lässt sich das mögliche Themenspektrum der Ausstellungsarbeit sehr breit aufstellen, zumal auch

8 mediengeschichte.dnb.de.

9 http://www.dnb.de/DE/Ausstellungen/Leipzig/leipzig_node.html. der Themenkreis und der gesetzliche Auftrag der Deutschen Nationalbibliothek, der seit 2006 neben der Sammlung und Bewahrung aller gedruckten Publikationen und der Musik auch die Speicherung von Netzpublikationen umfasst, zu den großen kulturellen Fragestellungen von Gedächtnisinstitutionen gehört.

Eine kleine Auswahl an Ausstellungsthemen mag die Intention des Programms beleuchten:

Die erste Ausstellung nach dem Umzug des Museums in den Erweiterungsbau der Deutschen Nationalbibliothek widmete sich 2013 der Frage, wie das Medium Plakat in dem von Medienumbrüchen gezeichneten 20. Jahrhundert für die neuen Medien geworben hat. Die Ausstellung stellte das Bildmedium Plakat also unter mediale Selbstbeobachtung. Anhand von ca. 100 Beispielen konnte gezeigt werden, wie sich Funktion und Bildsprache von Plakaten seit dem Aufkommen des Mediums am Ende des 19. Jahrhunderts verändert hat.

Die Kabinettausstellung „Vanitas. Tod im Buch“, die anlässlich des größten europäischen Treffens der „Schwarzen Szene“ - dem alljährlich zu Pfingsten in Leipzig stattfindenden „Wave Gotik Treffen“ - 2014 eröffnet wurde, thematisierte den vielgestaltigen Umgang von Typografen, Illustratoren und Buchgestaltern mit dem Thema Tod.

Der ersten (und letzten) Weltausstellung des Buches, der 1914 am Vorabend des Ersten Weltkrieges in Leipzig eröffneten „Internationalen Ausstellung für Buchgewerbe und Graphik“ (Bugra), widmete das Museum zum 100. Jahrestag eine Schau, die durch die zahllosen Anknüpfungspunkte an die Zeitgeschichte überraschte. ${ }^{10} \mathrm{Zu}$ Beginn des 20. Jahrhunderts als internationale Friedensmission geplant, versank die Weltausstellung, die auf große internationale Resonanz gestoßen war, mit dem Ausbruch des Ersten Weltkrieges im nationalistisch geprägten Kampf der Fronten dieses ersten Maschinenkrieges.

Die Kabinettausstellung „Kafka im Künstlerbuch“ widmete sich 2015 einem Thema, das einen Blick in einen wertvollen Bestand des Museums erlaubt: die Sammlung an Künstlerbüchern. Anlass der Ausstellung waren zwei Tage im Leben Franz Kafkas: Am 28. und 29. Juni 1912 begleitete der Schriftsteller Max Brod seinen Freund Kafka nach Leipzig, um in der Buchstadt einen Verleger für dessen Texte zu finden. Kafka tat sich zeitlebens schwer mit der Veröffentlichung seiner Texte, gerade die Frage nach der Bebilderung seiner Imaginationen war immer wieder Anlass zu Auseinandersetzungen mit seinen wenigen Verlegern. Umso erstaunlicher ist es zu sehen, wie viele Buchkünstler und Illustratoren in den letzten 100 Jahren mit

10 Fischer und Jacobs (2014). 
den Texten Kafkas gearbeitet haben. Die Ausstellung beschränkte sich angesichts der unüberschaubaren Fülle an buchkünstlerischen Bearbeitungen auf die wenigen Texte, die der von Selbstzweifeln geplagte Kafka zur Veröffentlichung freigegeben hatte.

Die Ausstellung „SchriftBild. Russische Avantgarde“"11 knüpfte 2015 an die zeitgenössische Schrift- und Bildgestaltung im öffentlichen Raum an und fragt nach ihren Ursprüngen. Dabei spielen die Künstler der russischen Avantgarde eine zentrale Rolle, die in den 20er-Jahren des 20. Jahrhunderts eine radikal neue, abstrakte Bildsprache erfanden. Als „Ohrfeige dem öffentlichen Geschmack“ schlug die Kunst der jungen Wilden in die Welt der Grafik, Malerei und Buchgestaltung ein. Noch heute zehrt das aktuelle Design von den Innovationen der Russischen Avantgardisten.

Die Kabinettausstellung „Reading a book? Hollywood liest“ widmete sich einem filmhistorischen Thema und spiegelte damit zugleich das Interesse des Museums an transmedialen Fragestellungen: Eine Sammlung von Fotos aus der Welt Hollywoods nimmt das Requisit Buch im Film unter die Lupe. Wie verändert sich dieses Requisit im Laufe der Filmgeschichte - begonnen in der Frühzeit des Films in den 20er-Jahren bis zum Ende des 20. Jahrhunderts.

Seit September 2016 schließlich nimmt das Deutsche Buch- und Schriftmuseum den 500. Jahrestag der Reformation zum Anlass, das Flugblatt als Medium in den Blick zu nehmen. Ohne das Flugblatt, das erst durch die Erfindung des Buchdrucks mit beweglichen Metalllettern möglich wurde, wäre die rasante Verbreitung der Ideen Martin Luthers nicht möglich gewesen. Doch verharrt die Ausstellung nicht in der frühen Neuzeit, sondern fragt nach Funktions- und Gestaltungswandeln der fliegenden Blätter bis heute. Ob im Syrienkrieg oder auf der sogenannten Balkanroute, ob bei Kundgebungen der Pegida-Bewegung oder beim Protest von Schülern gegen Bildungsmissstände: Das Flugblatt ist bis heute ein Medium der Information, des Protestes und der Propaganda.

11 Jacobs (2015).

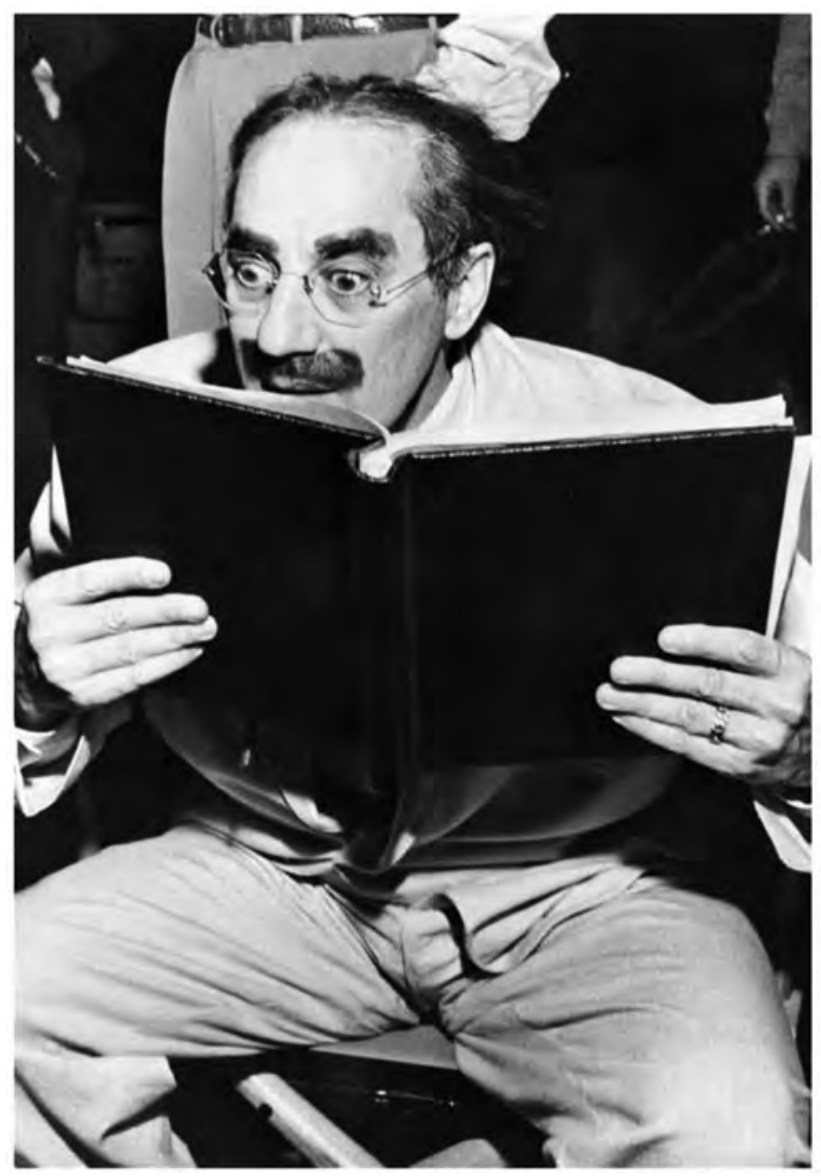

Abb. 7: Aus der Ausstellung „Reading a book? Hollywood liest“: Groucho Marx, Promotionsfoto von United Artists, $1945^{\circledR}$ Sammlung Günter Karl Bose

\section{Literaturverzeichnis}

Fabian, Bernhard (1994): Entwicklung des Deutschen Buch- und Schriftmuseums der Deutschen Bücherei Leipzig: Denkschrift. Leipzig: Die Bibliothek.

Fischer, Ernst; Jacobs, Stephanie (2014): Die Welt in Leipzig: Bugra 1914. Internationale Ausstellung für Buchgewerbe und Graphik 1914. Hamburg: Maximilian-Gesellschaft.

Funke, Fritz (1984): Das Deutsche Buch- und Schriftmuseum der Deutschen Bücherei in Leipzig. In: Gutenberg-Jahrbuch; begr. von A. Ruppel, 59, 194-210.

Güntner, Joachim (2012): Keilschrift und Binärcode. Neue Dauerausstellung im Buch- und Schriftmuseum Leipzig. In: Neue Zürcher Zeitung, (31. März 2012), 24.

Hintermeier, Hannes (2012): In den Wunderkammern des Wissens. Leipzig strahlt: Die neue Dauerausstellung des Deutschen Buchund Schriftmuseums. In: Frankfurter Allgemeine Zeitung, (17. März 2012), 31.

Jacobs, Stephanie (2014a): Restitution von Beutebüchern. In: Dialog mit Bibliotheken, 26 (1), 68-71.

Jacobs, Stephanie (2014b): Zeichen - Bücher - Netze: Von der Keilschrift zum Binärcode. Das Deutsche Buch- und Schriftmuseum 
im Erweiterungsbau der Deutschen Nationalbibliothek in Leipzig. In: Gutenberg-Jahrbuch: begr. von A. Ruppel, 89, 13-30.

Jacobs, Stephanie (Hrsg.) (2015): SchriftBild. Russische Avantgarde: Ausstellung im Deutschen Buch- und Schriftmuseum der Deutschen Nationalbibliothek. Leipzig, Frankfurt am Main: Deutsche Nationalbibliothek.

Jacobs, Stephanie (Hrsg.) (2016): Zeichen - Bücher - Netze: Von der Keilschrift zum Binärcode: ein Gang durch die Ausstellung. Göttingen: Wallstein Verlag.

Jacobs, Stephanie, Rüdiger, Bettina (2016): Buch - Museum - Zeitgeschichte: Die Bestände des Deutschen Buch- und Schriftmuseums der Deutschen Nationalbibliothek in Moskau. In: Durch Dialog zur Zusammenarbeit: Über den deutsch-russischen Bibliotheksdialog zu kriegsbedingt verlagerten Büchersammlungen, hg. v. Olaf Hamann, 111-15. Berlin: Staatsbibliothek zu Berlin Preußischer Kulturbesitz (Veröffentlichungen der OsteuropaAbteilung / Staatsbibliothek zu Berlin - Preußischer Kulturbesitz: Band 43).

Müller, Lothar (2012): Durch der Jahrhunderte Strom hin zum elektrischen Blatt. Das neu gemachte Deutsche Buch- und Schriftmuseum in Leipzig hütet das Wissen und fragt nach der Zukunft des Mediums. In: Süddeutsche Zeitung, (14. März 2012), 11.
Poethe, Lothar (1997): "Wo ist Gutenbergbibel?": Die wertvollsten Bestände des Deutschen Buch- und Schriftmuseums in Leipzig als Kriegsbeute. Ein Fallbeispiel zum Thema Trophäenliteratur, anstelle einer Rezension. In: Leipziger Jahrbuch zur Buchgeschichte, 7, 247-301.

Schmidt, Frieder (2016): Bahnriss?! Papier | Kultur. Eine Ausstellung im Deutschen Buch- und Schriftmuseum. In: Dialog mit Bibliotheken, (1), 42-47. Verfügbar unter http://d-nb.info/108253787 $\mathrm{x} / 34$.

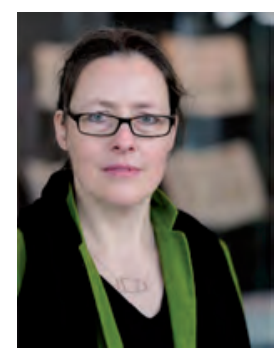

Dr. Stephanie Jacobs

Deutsche Nationalbibliothek

Leiterin Deutsches Buch- und Schrift-

museum

Deutscher Platz 1

D-04103 Leipzig

s.jacobs@dnb.de 